Meta

Journal des traducteurs

Translators' Journal

\title{
Normes, fonctions et traduction théâtrale
}

\section{Louise Ladouceur}

Volume 40, numéro 1, mars 1995

URI : https://id.erudit.org/iderudit/003371ar

DOI : https://doi.org/10.7202/003371ar

Aller au sommaire du numéro

Éditeur(s)

Les Presses de l'Université de Montréal

ISSN

0026-0452 (imprimé)

1492-1421 (numérique)

Découvrir la revue

Citer cet article

Ladouceur, L. (1995). Normes, fonctions et traduction théâtrale. Meta, 40(1),

31-38. https://doi.org/10.7202/003371ar d'utilisation que vous pouvez consulter en ligne.

https://apropos.erudit.org/fr/usagers/politique-dutilisation/ 


\section{NORMES, FONCTIONS ET TRADUCTION THEATTRALE}

LOUISE LADOUCEUR

University of British Colombia, Vancouver. Canada

L'article qui suit expose, dans ses grandes lignes, le cadre théorique adopté pour l'élaboration d'un mémoire de maîtrise déposé à l'Université de Montréal en 1991 sous le titre Les paramètres de l'adaptation théatrale au Quéhec de 1980 à 1990. Cette recherche, qui proposait de rendre compte des normes, des stratégies et des concepts translatifs dont procède l'adaptation de l'ouvre dramatique au Québec, s'est inspirée principalement de la méthodologie fonctionnaliste élaborée par Gideon Toury (1980).

Après avoir décrit les éléments théoriques qui ont fondé notre étude, nous proposons un modèle d'analyse descriptive approprié au texte dramatique puis nous traitons des rapports que la traduction théâtrale entretient avec le système littéraire qui l'accueille et l'ensemble socioculturel dont il fait partie.

\section{L'HYPOTHESE, FONCTIONNALISTE}

Inspirée des travaux théoriques d'Itamar Even-Zohar dans le domaine de la traduction littéraire, I'hypothèse fonctionnaliste exprimée par Gideon Toury s'inscrit dans la recherche d'une théorie à caractère sémiotique - c'est-à-dire qui tient compte du rôle joué par différents systèmes de signes dans l'acte de traduire - axée sur la fonction que le texte littéraire traduit est appelé à remplir dans la littérature d'arrivée. La grande originalité de cette recherche, orientée principalement sur l'étude du texte-cible contrairement à la plupart des théories déjà formulées, est de s'élaborer à partir de l'analyse descriptive systématique d'un maximum de traductions littéraires existantes. En cela, elle tranche nettement avec l'approche normative traditionnelle reposant sur des modeles de traductions hypothétiques qui débordent rarement le cadre du texte, voire de la phrase.

Cette étude descriptive de la traduction n'a donc plus pour objet de déterminer une façon idéale de traduire, mais de voir plutôt comment on traduit, à quelles modalités translatives est soumis le texte afin de pouvoir fonctionner dans la langue et la littérature d'accueil comme équivalence d'un texte d'une autre langue. appartenant à une autre littérature. De ce point de vue, toute analyse de la traduction doit nécessairement se rapporter à la fonction assignée à l'œuvre traduite dans son contexte adoptif. La traduction littéraire étant considérée comme un produit linguistique el textuel appartenant d'abord au système de la langue-cible. le système modélisant primaire, et ensuite au système littéraire récepteur, le secondary modeling system (Toury 1980: 37), qui lui servent de modèles, l'étude descriptive de la traduction doit comprendre non seulement celle du phénomène linguistique que représente le texte traduit, mais aussi celle du corps et du polysystème (Even-Zohar 1979) littéraires dont il fait partie.

\section{L'équivalence fonctionnelle}

Dans un tel contexte. l'équivalence n'est plus une notion absolue mais un phénomène empirique et observable. de nature fonctionnelle, où le rapport existant entre deux énoncés appartenant à des langues différentes fait en sorte que la traduction differe du texte original tout en le représentant au sein du milieu d'adoption. Bien que les deux 
textes s`apparentent aux mêmes traits pertinents ou relevant featuress (Toury 1980) : 38). la hiérarchie de ces traits diffère selon le point de vue du texte-cible et celui du texte-source, respectivement soumis à des contraintes systémiques distinctes.

Cette équivalence fonctionnelle d'une traduction littéraire se définit par sa position entre le pôle de l'adéquation au texte de départ, adéquày (Toury 1980: 55), et celui de son acceptabilité, acce'ptability (ibid.), dans les systèmes linguistiques et littératires d'arrivée. La position occupée par la traduction entre ces deux pôles est déterminée par des normes qui agissent comme facteur intermédiaire entre les équivalences virtuelles et le fonctionnement réel de certaines d'entre elles dans le texte. Le concept d'équivalence est ainsi gouverné par les normes qui modèlent la traduction de façon à ce qu elle soit une auvre valable dans la langue-cible el qu elle occupe une position appropriée dans le polysystème littéraire visé. tout en représentant un lexte d'une autre langue occupant lui-même une position dans un autre polysystème littéraire. Ces déux éléments essentiellèment différents sont souvent incompatibles.

\section{Les normes}

Dans une société donnée. la manière de traduire est façonnée par les contraintes modelant à des degrés divers le comportement des individus qui appartiennent à cette société et en partagent les valeurs et les idées. Ces règles explicites ou implicites, apprises au cours du processus de socialisation, constituent ce qu en termes sociologiques on appelle des norme's. De nature intersubjective. les normes occupent une section d un continuum allant d'un extrême objectif, représenté par les lois formulés, à un extrême subjectif, qui se manifeste par idiosyncrasies. Formant un ensemble propre à chayue communauté linguistique el littéraire, les normes agissent à toutes les étapes du processus translatif et à tous les niveaux de production de la traduction.

Bien qu'elles soient de nature et d'intensité variées, les normes traductionnelles participent toutes d'une norme initiale, inirial norm (Toury 1980: 54). Cette norme des normes détermine la valeur de la traduction, c'est-à-dire la priorité accordée par le traducteur à la recherche d'adéquation (l'adhérence au texte-source et à sés normes) ou à la recherche d'acceptabilité (l'adhérence aux normes actives dans les systèmes linguistique et littéraire d'arrivée). Dans la pratique, ce choix de basé, cette approche globale de la traduction d'un texle particulier adoptée par un traducteur spécificue prendra la forme d'un compromis entre les deux extrêmes en polarité, d'une rencontre ou d'une confrontation entre deux ensembles de signes.

Les normes Iraductionnelles se divisent en deux groupes majeurs, respectivement issus des deux questions centrales posées par l'hypothèse fonctionnaliste : quelles ceuvres choisit-on de traduire et comment les traduit-on? Ou, plus précisément, comment le polysystème récepteur régit-il le choix du texte à traduire et la façon de le traduire? Présidant à la sélection des textes à traduire, les normes préliminaires, preliminary norms (Toury 1980: 53), concernent la politique de traduction adoptée, c'est-à-dire les facteurs systématiques reliés au choix des ceuvres, des auteurs, des traducteurs. des genres privilégiés ou ignorés par la traduction el l'utilisation de versions intermédiaires dans le processus de traduction. De leur côté. les normes opérationmelles, operational norms (Toury 1980: 54), orientent les décisions faites au moment de la traduction. Celles-ci se divisent en deux genres: de nature matricielle. elles se rapportent à l'existence même du matériau linguistique-cible pouvant servir de substitut au matériau linguistique-source, ì sa distribution et à sa segmentation dans le texte; de nature texmelle, elles se rapportent au choix exercé sur ce matériau en remplacement du texte original. Ce choix est linguistique lorsqu il est relié, par exemple, aux normes stylistiques propres à une langue donnée ; il est littéraire lorsqu il obéit à ce qui est perçu comme approprié pour une a'uvre littéraire ou une traduction selon le lieu, la période, le genre ou la technique utilisée. 
Au niveau de l'intensité, une réinterprétation du modèle de Jay Jackson (Jackson 1965 : 303) permet de procéder à une division tripartite des normes (figure I). Celles-ci sont qualifiées de primaires lorsque, situées au sommet de la courbe, elles représentent un phénomène plus ou moins obligatoire et offrent un minimum de latitude au traducteur; elles sont secondaires et occupent la partie la plus rapprochée du sommet lorsqu'elles expriment des tendances acceptables, généralement admises par le groupe étudié: enfin. elles deviennent des comportements tolérés lorsque. situées dans la partie inférieure de la courbe, elles offrent le maximum de latitude au traducteur. La dernière catégorie comprend en outre les formules symptomatiques caractéristiques du groupe étudié. Cependant, il ne faut pas confondre les normes avec les universaux du comportement traductionnel. comme la tendance à rendre explicite dans la traduction ce qui est implicite dans le texte original.

FIGURE 1

Représentation graphique des normes

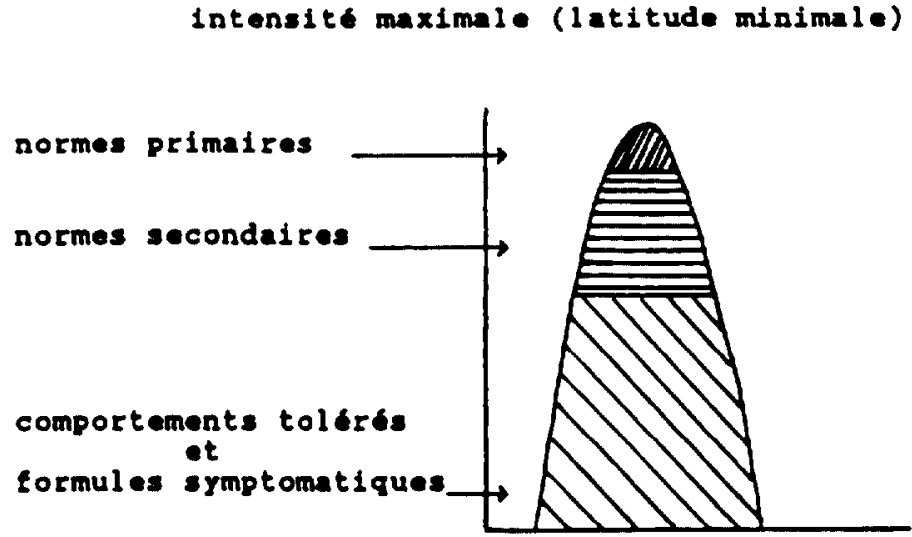

L'étude des normes traductionnelles puise à des sources textuelles. soit les textes traduits et leur inventaire analytique. ainsi qu'à des sources extratextuelles telles que les formulations critiques ou semi-théoriques, les commentaires, les analyses et les exposés portant sur la traduction. Les premières permettent de reconstruire les normes, les secondes contiennent des normes déjà formulées. L'identification des normes textuelles se fait à partir d'une recherche méthodologique des plus petites unités de normes, les normèmes (Toury 1980: 59), laquelle s'effectue de l'axe paradigmatique à l'axe syntagmatique vers un regroupement cohérent des conclusions tirées de l'analyse de tous les normèmes identifiés.

\section{La traduction adéquate}

La comparaison entre le texte original et sa traduction étant unilatérale et irréversible puisqu'elle n'agit d'aucune façon sur le texte de départ, c'est celui-ci qui sert de base à l'invariant de la comparaison : la traduction adéquate, adequate translation (Toury 1980: 112), construction intermédiaire hypothétique contenant le maximum d'équivalence. Ainsi, l'objet de la comparaison est d'établir la distance existant entre l'équivalence 
texte-cible/texte-source et celle de l'adéquation maximale au texte-source. représentée par la notion idéale de traduction adéquate.

Le texte-source étant structuré d'après des codes linguistiques eux-mêmes modelés par des codes littéraires, il s'y établit un réseau complexe de relations textuelles dont les éléments possèdent des fonctions précises à l'intérieur du texte. L'identification de ces éléments textuels, les textèmes (Even-Zohar 1974 in Toury 1980: 114), et plus particulièrement celle des textèmes-jonction qui sont liés à plusieurs fonctions textuelles, à différents éléments microstructurels ou à divers principes agissant sur le texte. est indispensable à la formulation de la traduction adéquate à partir de laquelle s'effectue la comparaison des unités du texte-cible correspondant aux textèmes du texte-source. Cette comparaison permet de relever les écarts entre l'équivalence idéale de la traduction adéquate et celle existant entre le texte de départ et sa traduction.

\section{MODELLE. D'ANALYSE, DESC 'RIPTIVE}

Depuis le milieu des années soixante-dix, des universitaires de différents pays travaillent en étroite collaboration à l'élaboration d'un nouveau paradigme d'étude de la traduction littéraire, fondé sur une théorie d'ensemble qui tiendrait compte du plus grand nombre d'éléments intervenant dans le processus translatif et des recherches effectuées sur la pratique même de la traduction. Considérant la littérature comme un système dynamique complexe. et convaincus de l'action réciproque que doivent exercer l'un sur l'autre les modèles théoriques et les textes existants, les membres de ce groupe préconisent une approche de la traduction de nature descriptive, systémique et sémiotique, axée sur une notion fonctionnelle d'équivalence et orientée vers l'étude du texte-cible. Ils s'intéressent aussi aux normes et aux contraintes qui gouvernent la production et la réception d'un texte, aux relations entre traduction et autres catégories de texte ainsi qu'au rôle joué par la traduction dans une littérature donnée ou dans des littératures en interaction (Hermans $85: 10-11$ ).

Parmi ces chercheurs. José Lambert et Hendrik van Giorp, qui ont aussi élaboré un modèle de communication global de la traduction selon les paramètres correspondant aux phénomènes traductionnels relevés par Itamar Even-Zohar et Gideon Toury, reconnaissent le rôle essentiel de la comparaison de textes dans l'étude de la traduction, mais en déplorent l'aspect trop souvent réductionniste. Ils réprouvent le recours unique au texte de départ comme cadre de référence servant à la comparaison des textes car. selon eux. cette pratique n'aurait pour effet que de montrer ce que la traduction n'est pas. Il est à noter que l'utilisation de la traduction adéquate comme cadre de référence présente en outre le désavantage de restreindre la comparaison au seul binôme texte-source/texte-cible en cause. Jugeant donc cette approche différentielle négative et unidirectionnelle, Lambert et van Gorp proposent comme outil de référence un modèle hypothétique complexe applicable indifféremment à chaque texte éludié et permettant d'identifier non seulement les traits caractéristiques du texte, mais aussi les stralégies qui y sont à l'ceuvre, leur ordre de priorité et la nature des normes dont elles relèvent.

Fondé sur une hypothèse de correspondance entre les mécanismes translatifs ceuvrant à différents niveaux de structure textuelle, ce modèle préconise une étude télescopique des stratégies de traduction allant de la présentation générale du texte, où se devine déjà une stratégie d'ensemble, à sa macrostructure et, enfïn, aux stratégies microstructurelles. Un tel modèle permet une classification des phénomènes observés selon des paramètres spécifiques et facilite, en fin d'analyse, leur confrontation avec le contexte systémique dans lequel ils šinscrivent. Celle approche. essentiellement systémique puisqu'elle n’éludie le phénomène translatif qu'en rapport avec le système qui l'englobe. à savoir tous les textes traduits ou écrits qui participent à la formation d'une littérature, permet en 
outre d'évaluer le caractère individuel ou collectif d'une norme selon la fréquence des phénomènes translatifs qui lui sont attribuables.

Inspiré du tableau synthétique élaboré par José Lambert et Hendrik van Gorp (1985: 52) pour la description de la traduction, tableau que nous avons redéfini en fonction des particularités de l'cuurre dramatique. le modèle suivant propose un plan d'analyse descriptive adapté au texte de théâtre. Pour les raisons exprimées précédemment. ce modèle, présenté sous forme synthétique dans le tableau qui suit. a été préféré au concept de traduction adéquate comme cadre de référence pour l'analyse et la comparaison des textes dramatiques en traduction.

\section{Modele d'analyse descriptive}

1. Données préliminaires

a) Présentation du texte

- titre de la traduction:

- nom du traducteur:

- titre original de la piecce

- nom de l'auteur:

- indications de genre:

- date. lieu. équipe de production.

b) Métatextes

- pages liminaires:

- préface:

- notes incluses ou séparées :

- notes de bas de page.

c) Indications de stratégie

- complète:

Iranslative

- partielle:

générale

- amplifiée.

(Hypothèses relatives à la macrostructure)

2. Analyse de la macrostructure

a) Encadrement du texte

- prologue:

- épilogue.

b) Divisions du texte

- actes el scènes:

- titres et divisions.

c) Indications scéniques

- personnages;

- lieux:

- époyue:

- décors el accessoires ;

- costumes:

- description de l'action :

- didascalies.

(Hypothèses relatives aux microstructures)

3. Analyse des microstructures

a) Niveaux de langue

- littéraire, soutenu, courant, familier, populaire, argotique :

b) Modèles grammaticaux

- dialectes. particuliers

- élisions, répétitions,

- inversions, omissions, etc.

c) Vocabulaire

- écarts graphiques:

- ecarts lexico-sémantiques. 
d) Procédés stylistiques particuliers

c) Ecarts sémantiques
- modes narratifs:

- figures de styles.

- ajouts:

- suppressions;

- substitutions:

- autres modifications.

(Mise en rapport des résultats de chaque niveau d’analyse et confrontation avec le contexte systémique)

4. Confrontation aree le contexte sustémicue

a) Comparaison

b) Relations intertextuclles

c) Relations inter-systémiques
- textes traduits:

- données extratextuelles.

- autres traductions du même auteur:

- créations du même aluteur.

- structures des differents genres

- specificités stylistiques.

\section{LA TRADUCTION ETHNOCENTRIQUE. PORTEUSE DU DISCOURS SOCIAL,}

Selon Antoine Berman, la traduction en Occident est soumise depuis la Rome antique à deux principes dominants. l'ethnocentrisme el l'hypertextualité. Traduction ethnocentrique signific:

qui ramène tout à sa propre culture, à ses normes et valeurs, et considere ce qui est silué en dehors de celle-ci - l'Ftranger - comme négatif ou tout juste bon à être annexé, adapté. pour accroître la richesse de celte culture. (Berman 1985: 48-49)

et traduction hypertextuelle renvoie à :

tout texte s'engendrant par imitation, parodie, pastiche, adaptation, plagiat, ou toute autre espèce de transformation formellè à partir d'un autre texte déjà existant. (Berman 1985:49)

Ces deux principes sont indissociables.

Déjà. l'impératif de transparence exigeant de la traduction qu elle "se lise comme un original» (Horguelin 1985: 82) et la primauté accordée au sens'. concept voulant qu' «une fois le sens saisi, il /doive| être restitué en fonction des idées et non en fonction des mots" (Delisle 1980: 82), ont conféré à la langue d'arrivée une valeur d’irréductibilité à la langue de départ qui oblige à une acclimatation du sens de l'auvre étrangère de telle sorte que cette dernière «apparaisse comme un fruit de la langue propre» (Berman $1985: 53$ ). Il y a donc par effet de modélisation aux normes matricielles du système linguistique de la langue-cible - le système modélisant primaire - réaménagement du sens de l'ceuvre étrangère selon les ressources et l'économic propres à la langue d'arrivée.

En outre. si l'on considère la littérature comme une formation discursive participant au discours social, cet «ensemble des ensembles" (Belleau 1981: 15) formé de:

tout ce qui se dit et s'écrit dans un étal de société. dans la mesure où cet ensemble $n$ 'apparaît pas comme composé d'énoncés aléatoires, mais au contraire. réglés par des conventions, pris dans des configurations idéologiques, (Angenot 1979: 6.3)

la traduction littéraire en tant que composante d un polysystème littéraire «enchâssé dans les structures idéologiques et socio-économiques de la société" (Hermans 198.5: 11) est essentiellement tributaire de slordre du discours" (Foucault 1971) en vigueur dans cette société. Voici comment Michel Foucault définit la pratique discursive: 
un ensemble de règles anonymes, historiques, toujours déterminées dans le temps et l'espace qui ont défini à une époque donnée, et pour une aire sociale donnée, les conditions d'exercice de la fonction énonciative. (Foucault 1969: 154)

Ainsi chargée de transmettre les valeurs de la société d'accueil, la traduction est soumise à diverses manipulations qui ont pour objet de la rendre «acceptable, c'est-à-dire conforme à l'ensemble des codes qui régissent à des degrés divers le discours de la société-cible» (Brisset 1990: 26). En ce sens, traduire est un acte discursif doté d'une "fonction doxologique» (Brisset 1990: 312) qui agit sur les relations d'équivalence virtuelles suggérées par le texte de départ.

Dans son étude sur les codes de la traduction théâtrale pratiquée au Québec entre 1968 et 1988. Annie Brisset soutient que cette fonction doxologique de la traduction fait en sorte qu'au Québec, à une époque d'effervescence nationaliste, traduire le théâtre :

n'a plus pour objet de transmettre le discours de l'Étranger mais d'utiliser l'Étranger pour cautionner son propre discours, celui de l'émancipation nationale. (Brisset 1990: 312)

C'est ainsi que, dans une société désireuse d'affirmer son identité nationale, désir dont un des corollaires discursifs est «'éclipse de l'altérité» (ibid.: 311), le texte étranger est devenu «miroir dans lequel on cherche sa propre image" (ibid.: 312). Modelée par les codes propres au système littéraire de la société réceptrice - le secondary modeling system de Toury, concept apparenté d'ailleurs au système modélisant secondaire que Juri Lotman définit ainsi :

un système de signes et de règles de combinaison propres à la littérature qui se superposent à la langue naturelle et servent à transmettre des informations particulières, non transmissibles par d'autres moyens (Lotman 1973: 52)

la traduction doit réaménager le discours étranger selon les règles d'acceptabilité du discours en place et se faire, à son tour, porteuse de ce discours. Dans cette foulée, l'adaptation. modalité translative où l'ethnocentrisme se manifeste avec le plus d'intensité, aura fréquemment recours à des stratégies visant à effacer les marques de l’Étranger pour donner à l'ceuvre dramatique une apparence locale.

C'est donc en suivant le modèle d'analyse descriptive présenté plus haut et en tenant compte des deux systèmes modélisants qui façonnent la traduction selon les normes matricielles et textuelles de la langue d'arrivée et selon les codes littéraires et discursifs en vigueur dans le contexte réceptif que nous avons par la suite tenté de dresser un tableau des paramètres de l'adaptation dans les textes dramatiques retenus pour notre étude.

Il serait trop long de commenter en détail dans cet article les résultats obtenus. Ce qui a surtout retenu notre attention, après la mise à découvert des phénomènes translatifs à l'œuvre dans les adaptations et les traductions qui composaient le corpus, c'est le fait qu'aucune des stratégies dont se réclame l'adaptation ne semble lui appartenir en propre. En effet, contre toute attente, l'analyse a révélé que les textes traduits et les textes adaptés font appel à des stratégies translatives de même nature, mais à des fréquences et à des degrés variés. Ainsi, loin de se constituer en mode translatif qualitativement distinct, l'adaptation se caractériserait plutôt de façon quantitative par un recours plus fréquent à certains procédés translatifs qui ne lui sont toutefois pas spécifiques.

Notes

1. Sur la primaute du sens, voir, entre autres, les travaux de Eugene Nida. Danica Seleskovitch et Maurice Pergnier. 


\section{REFERENCES}

ANGENOT, Marc (1979) : Glessaire pratique de la critique contemporaine. Montréal, Hurtubise.

BELL.FAU, André (1981): “le conflit des codes dans l'institution littéraire québecoise", liherté, $n^{\circ}$ 1.34, marsavril.

BERMAN. Antoine (198.5): "La traduction et la lettre ou l'auberge du lointain", Les Tours de Babel, Gérard Granel (dir.), Mauvezin. Editions Trans-Europ-Repress.

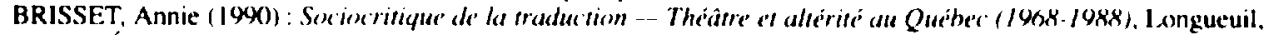
Les f́ditions du Préambule.

DELISLE, Jean (1980) : L analyse du discours comme méthode de traduriom. Otawa. Presses de l'l Iniversité d'Ottawa.

EVEN-ZOHAR, Jamar (1479): "Polysystem Theory". Poetics Todas, vol. I.

FOUCAULT, Michel (1969): L archéplogle du survir, Paris, Ciallimard.

FOUCAULT, Michel (1971): L'ordre de discours. Paris. Gallimard.

HERMANS. Theo (1985): "Translation Studies and a New Paradigm". The Manipulamon of liferofure, Theo Hermans (Ed.). London, Croom Helm.

HORGUELIN, Paul (198.5): Pratique de la révision, Montreal, Linguatech.

JACKSON, Jay (1965): "Structural Characteristics of Norms". Current Studies in Social Psvehologs, Steiner \& Fishbein (Fd.). New York. Holt, Rinehart \& Winston.

LAMBERT Jose el Hendirk VAN (iORP (I9X.5): "On Describing Translations", The Mampularion of Literature. Theo Hermans (Ed.), London, ('room Helm.

LOTMAN, Juri (1973): La structure du rewle arsishluwe, Paris, (jallimard.

TOURY, Gideon (1980): In Search of a Theory of Translatmon, Tel Aviv, The Ponter Institute for Poetics and Semiotics. 